\title{
Numerical studies of turbulent particle-laden jets using spatial approach of one-dimensional turbulence
}

\author{
Marco Fistler*1, David Lignell ${ }^{2}$, Alan Kerstein ${ }^{3}$ and Michael Oevermann ${ }^{1}$ \\ ${ }^{1}$ Division of Combustion, Chalmers University of Technology, Sweden \\ ${ }^{2}$ Brigham Young University, Provo, UT 84602, USA \\ ${ }^{3}$ Consultant, 72 Lomitas Road, Danville, CA 94526, USA \\ ${ }^{*}$ Corresponding author: marco.fistler@chalmers.se
}

\begin{abstract}
To challenge one of the major problems for multiphase flow simulations, namely computational costs, a dimensionreduced model is used with the goal to predict these types of flow more efficiently. One-dimensional turbulence (ODT) is a stochastic model simulating turbulent flow evolution along a notional one-dimensional line of sight by applying instantaneous maps that represent the effect of individual turbulent eddies on property fields. As the particle volume fraction is in an intermediate range above $10^{-5}$ for dilute flows and under $10^{-2}$ for dense ones, turbulence modulation is important and can be sufficiently resolved with a two-way coupling approach, which means the particle phase influences the fluid phase and vice versa. For the coupling mechanism the ODT multiphase model is extended to consider momentum transfer and energy in the deterministic evolution and momentum transfer during the particle-eddy interaction. The changes of the streamwise velocity profiles caused by different solid particle loadings are compared with experimental data as a function of radial position. Additionally, streamwise developments of axial RMS and mean gas velocities along the centerline are evaluated as functions of axial position. To achieve comparable results, the spatial approach of ODT in cylindrical coordinates is used here. The investigated jet configuration features a nozzle diameter of $14.22 \mathrm{~cm}$ and a Reynolds number of 8400 , which leads to a centerline inlet velocity of $11.7 \mathrm{~m} / \mathrm{s}$. The particles used are glass beads with a density of $2500 \mathrm{~kg} / \mathrm{m}^{3}$. Two different particle diameters $(25$ and $70 \mu \mathrm{m})$ were tested for an evaluation of the models capability to capture the impact of a varying Stokes number and also two different particle solid loadings ( 0.5 and 1.0$)$ were evaluated. It is shown that the model is capable of capturing turbulence modulation of particles in a round jet.
\end{abstract}

\section{Keywords}

Jet, particle-laden flows, turbulence modulation, one-dimensional turbulence

\section{Introduction}

Turbulent particle-laden jets play a major role in a wide range of industrial applications and natural phenomena. Especially the effect of particles on the gas-phase, named turbulence modulation, is of particular interest. Several experimental studies, e.g. Schreck and Kleis [11], Geiss et al. [4] and Budilarto [1], have shown the large influence of high particle concentrations on the turbulence level. To investigate this type of flow experimentally and to understand the physical fundamentals is still very challenging, because only a few advanced techniques to obtain spatially resolved unsteady data exist today. CFD (computational fluid dynamics) proved to be a powerful tool to investigate particle-laden flows and to acquire detailed flow information. Many CFD approaches for these flow types have limited predictive capabilities or rely on many assumptions, which affects the accuracy and restrict that generality. Even with access to a high-performance computational infrastructure direct numerical simulation (DNS) studies for particle-laden flow are still limited to academic cases and low particle numbers. On the other hand, the accuracy of large eddy simulations (LES) seems to be very sensitive to its parameters, especially of its subgrid-model, which is required to achieve a good turbulence prediction.

In our previous study [3] we achieved promising results with a stochastic particle dispersion simulation in a turbulent jet with an alternative approach called one-dimensional turbulence (ODT). ODT is a stochastic approach with reduced dimension to resolve the full range of length and time scales as in DNS and introduced in [5] and extended in [6]. ODT has demonstrated to predict topologically simple flows such as boundary layers and jets with large property gradients in one direction very well compared to DNS studies and experimental results. While molecular phenomena like viscous dissipation evolve along that single spatial dimension, the turbulent advection is modeled through a stochastic remapping of the velocity profiles, called eddy events. This and the previous study are a continuation of the work of Schmidt et al. [9] and Sun et al. [12], who extended the ODT model to predict particle-carrier phase interaction excluding particle collision. These models has still issues to convert temporal ODT output data to spatial results, which can be evaluated with spatial experimental results. Therefore, the model is extended by using a spatial approach of ODT in cylindrical coordinates. Also, a new formulation of the particle-eddy interaction is introduced. The following study is based on the same test cases as in [12], which includes experimental results from [1]. 


\section{One-dimensional turbulence}

This section describes the concept of the ODT model, which is used in this study to simulate the carrier gas phase. ODT is a stochastic model to simulate turbulent flows on a one-dimensional domain, which is usually oriented in the direction of the largest expected velocity gradients. In case of turbulent dispersed jet configurations the domain is oriented in radial/lateral direction $r$. By the assumption that a jet flow is axisymmetric, one realization with the radial coordinate $r$ plus the evolving direction $z$ can interpreted as a representation of a 3D unsteady flow field. For a spatial evolution of the ODT line in the Lagrangian framework the governing equations are described by a mass flux and a momentum flux balance (illustrated in Fig. 1). In ODT these equations are only governing the steady solution of the flow and the unsteadiness due to turbulence is captured by a random sequence of eddy events. In fact the model conserves statistically the steady solution of flows but also represents the unsteadiness of turbulence.
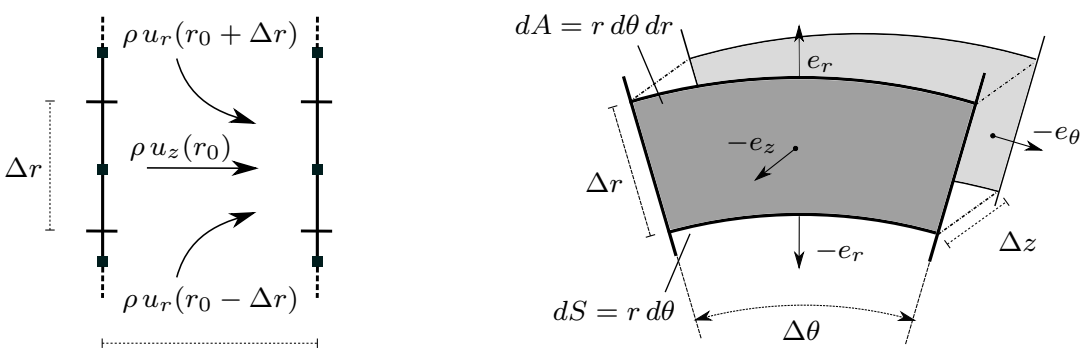

$\Delta z$

Figure 1. Sketch of mass flux balance and control volume.

\section{GOVERNING EQUATIONS}

Governing equations of spatial ODT evolution with a constant density over the domain are obtained by considering the balances of mass and momentum flux to a control volume (see Fig. 1). We consider an axisymmetric flow without swirl, i.e. $u_{\theta}=0$, and all quantities are independent of $\theta$. Therefore, the integration can be solved explicitly and the angle $\Delta \theta$ drops out. This shows that the mass flux balance is independent of $\theta$ and given as

$$
\int_{\Delta r} \frac{\partial\left(\rho u_{z}(r, z)\right)}{\partial z} r d r+\int_{\Delta z} \frac{\partial\left(\rho u_{r}(r, z)\right)}{\partial r} r d z=d_{\rho u_{z}}
$$

where $d_{\rho u_{z}}$ represents the sudden changes during eddy events in a spatial step $\Delta z$, which are discussed below in more detail. Outside the eddy region the term is zero. The same assumptions as above are applied to the momentum flux balance equation, which is given as

$$
\int_{\Delta r} \frac{\partial\left(\rho u_{z}(r, z) u_{i}(r, z)\right.}{\partial z} r d r+\int_{\Delta z} \frac{\partial\left(\rho u_{r}(r, z) u_{i}(r, z)\right.}{\partial r} r d z=\mu \int_{\Delta z} \frac{\partial\left(u_{i}(r, z)\right)}{\partial r} r d z+d_{\rho u_{z} u_{i}}+s_{p, i},
$$

where the right hand side summarizes all source and sink terms considered. The first represents viscous effects and the second, $d_{\rho u_{z} u_{i}}$, accounts for momentum transfer during eddy events in a spatial step $\Delta z . s_{p, i}$ describes the momentum transfer between dispersed and gas phase and is defined as

$$
s_{p, i}=\frac{1}{\int_{\Delta r} \rho u_{z} r d r} \sum_{j}^{n_{p}} m_{p, j}\left(u_{j, i}\left(r, z_{0}\right)-u_{j, i}\left(r, z_{0}+\Delta z\right)\right) .
$$

For this study the pressure term is omitted due to a zero pressure gradient in the flow configuration.

\section{EDDY EVENTS}

In ODT flow unsteadiness due to turbulence advection is modeled through eddy events, which results in remapping of the velocity profiles over a sampled eddy region $r_{0}<r<r_{0}+l$, characterized by a position $r_{0}$ and a length scale $l$. Both parameters are sampled randomly to mimic the occurrence of eddies in turbulent flow. The mapping method used in ODT is called triplet map [5]. In a planar reference frame it means the original profile is compressed by a factor of three over the eddy region and three copies are filled in. To ensure continuity of the profile the second copy in the middle is inverted. In planar ODT the cell sizes depend only on the length in ODT line direction. However, in a cylindrical framework it depends on the square of the length. As a result, in cylindrical coordinates the mapping process compresses the profiles with respect to the square of the length. The mapping function depending on the post-position for $r_{0} \geq 0$ is given as

$$
f(r)=r_{0}+ \begin{cases}\sqrt{3\left(r-r_{0}\right)^{2}} & \text { if } r_{0} \leq r \leq r_{0}+\sqrt{\frac{l^{2}}{3}} \\ \sqrt{2 l^{2}-3\left(r-r_{0}\right)^{2}} & \text { if } \sqrt{\frac{l^{2}}{3}} \leq r \leq \sqrt{\frac{2 l^{2}}{3}} \\ \sqrt{3\left(r-r_{0}\right)^{2}-2 l^{2}} & \text { if } \sqrt{\frac{2 l^{2}}{3}} \leq r \leq l \\ r-r_{0} & \text { otherwise. }\end{cases}
$$


For the case $r_{0}<0$ the algebraic signs have to be adjusted in consideration of the possibility that $r_{0}+l$ is greater or smaller zero. Examples are illustrated in Fig. 2.

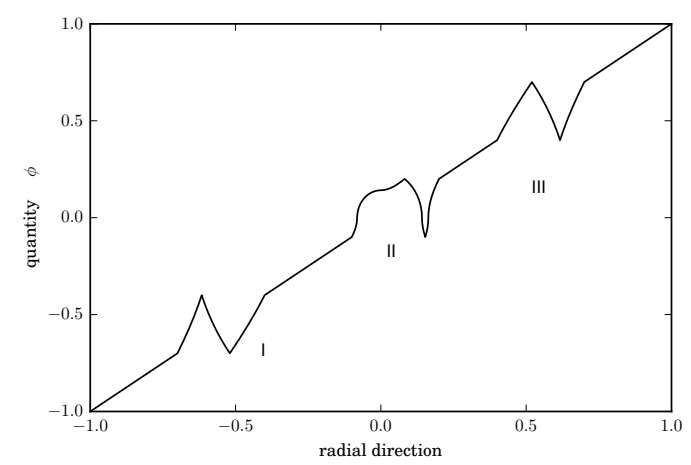

Figure 2. Examples for applying a cylindrical triplet map to a linear profile of quantity $\phi$. I: $r_{0}, r_{0}+l<0, \quad$ II: $r_{0}<0, r_{0}+l>0$, III: $r_{0}, r_{0}+l>0$, where $r_{0}$ and $l$ are eddy position and length, respectively.

An essential part of turbulence is the phenomena of return-to-isotropy, which requires on the ODT modeling side a re-distribution of turbulent kinetic energy among the velocity components. This is achieved by introducing kernel transformations to the mapping function, which gives a profile transformation

$$
u_{i}(y) \rightarrow u_{i}^{\mathrm{TM}}(y)+c_{i} K(y)+b_{i} J(y),
$$

where $u_{i}$ is the velocity in $i$-th direction before and $u_{i}^{\mathrm{TM}}$ after the mapping process. The Kernel $K(y)$ is defined as the fluid displacement profile under the triplet map and integrates to zero over the eddy region. $J(y)$ is the absolute of $K(y)$ and so it does not integrate to zero over region. Thus, it forces momentum change of the profiles if its kernel coefficient $b_{i}$ is non-zero. $c_{i}$ defines the kernel amplitude of $K(y)$. Thus, both kernels are important in the case of particle-gas phase coupling. Due to momentum and energy flux conservation over the sampled eddy region it is required to meet the following equations:

$$
\begin{aligned}
& \int \dot{\rho} u_{i} r d r=\int \dot{\rho}\left(u_{i}^{\mathrm{TM}}+c_{i} K+b_{i} J\right) r d r+S_{\mathrm{p}, i}, \\
& \frac{1}{2} \int \dot{\rho} u_{i}^{2} r d r=\frac{1}{2} \int \dot{\rho}\left(u_{i}^{\mathrm{TM}}+c_{i} K+b_{i} J\right)^{2} r d r-\Delta E_{i}+S_{\mathrm{pE}, i} .
\end{aligned}
$$

$S_{\mathrm{p}, i}$ and $S_{\mathrm{pE}, i}$ represent the sum of momentum and energy flux penalties caused by particles, respectively. $\triangle E_{i}$ stands for the above mentioned re-distribution of energy between velocity components. Under consideration of the measure preserving character of the mapping process itself, which means $\int \rho u_{i} r d r=\int \rho^{\prime} u_{i}^{\mathrm{TM}} r d r$ and $\int \rho u_{i}^{2} r d r=\int \rho^{\prime}\left(u_{i}^{\mathrm{TM}}\right)^{2} r d r, b_{i}$ and $c_{i}$ can be determined and define the new profile. Subsequently, the resultant kinetic energy of the sampled region is used to determine the eddy timescale $t_{\mathrm{e}}\left(l, r_{0}\right)$. Based on the scaling assumption for the kinetic energy $E \sim \rho l^{3} / 2 t_{\mathrm{e}}^{2}$, the eddy time scale is modeled as

$$
\frac{1}{t_{\mathrm{e}}}=C \sqrt{\frac{2}{\rho l^{3}}\left(E_{\mathrm{kin}}-Z E_{\mathrm{vp}}\right)} .
$$

The viscous energy penalty is given as $E_{\mathrm{vp}}=\mu^{2} / 2 \rho l$ and $C$ is the adjustable eddy rate parameter, which scales the overall eddy event frequency. $Z$ is the viscous penalty parameter, which suppresses unphysically small eddies. An equivalent procedure for large eddies exists as well. In this study the elapsed time method is used in which the eddy time can be compared with elapsed time $t$ of the simulation. Eddy events are only allowed if $t \geq \beta t_{\mathrm{e}}$, where $\beta$ is a model parameter.

We assume that the occurrence of eddies of a certain size follows a Poisson process in time with a rate determined by the eddy timescale provided in (8). Technically this is solved by oversampling, i.e. generation of candidate eddies at a much higher rate than requested, and thinning of the Poisson process with an acceptance-rejection method. For details we refer to [7]. 


\section{Lagrangian Particle Model}

The particulate phase is modelled in a Lagrangian way where individual particles are tracked following Newton's second law of motion. Here we consider only drag forces and gravity acting on the particles leading to the following set of equations:

$$
\begin{aligned}
& \frac{d u_{p, i}}{d t}=-\frac{u_{p, i}-u_{g, i}}{\tau_{p}} f+g_{i}, \\
& \frac{d r_{p}}{d t}=u_{p, r} .
\end{aligned}
$$

Here, the subscript $p$ and $g$ represent the particle and gas phase, respectively, and $g_{i}$ is the $i$-th component of the gravity acceleration vector. The particle response time, $\tau_{p}=\rho_{p} d_{p}^{2} / 18 \mu$ based on Stokes flow, is given with consideration of mass $m_{p}$ and density $\rho_{p}$ of the particle and the fluid viscosity $\mu$. Clift et al.[2] suggested a nonlinear correction factor $f$ for a particle slip-velocity Reynolds number $R e=\frac{\rho_{g}\left|\vec{u}_{p}-\vec{u}_{g}\right| d_{p}}{\mu}$ smaller than 200, which is for many practical dilute flow systems the case. The factor $f$ is defined as

$$
f=1+0.15 R e_{p}^{0.687} \text {. }
$$

The drag law (9) is solved by a first-order Euler method. As the ODT line evolves in spatial dimension $(\Delta z)$ the temporal step $\Delta t$ should be transformed. Therefore, a constant particle velocity over $\Delta z$ is assumed, which yields to

$$
\Delta t=\frac{\Delta z}{u_{p, z}} .
$$

In the spatial advancement of the steady-state solution of the underlying flow each particle always has a local gas velocity, which it interacts with. However, an eddy event appears instantaneous in the ODT simulation and so a model is required to capture the displacement in radial direction during a particle-eddy interaction.

\section{PARTICLE-EDDY INTERACTION MODEL}

The particle-eddy interaction (PEI) model is defined as the only effect of particle phase motion in ODT line-direction and so the gas velocity in this direction for the drag law (9) is zero. The PEI model in this study was developed by Schmidt et al. $[9,10]$ as a so-called instantaneous PEI model (noted as type-I) and governs the radial displacement due to an eddy event. Each particle obeys the model if they are located in the sampled eddy region. The main model assumption is that the eddy time scale $t_{\mathrm{e}}$ calculated in (8) defines the time an eddy needs to create the remapped profile. That means before an eddy event the particle motion in (9) is integrated over the eddy time with a radial velocity, which has to be corrected to account for the finite time of an eddy event. Therefore, it is required to define an eddy gas phase velocity in radial direction and an interaction time $\tau_{\mathrm{PEI}}$, which determines the time interval in (9), to correct the integration over the time interval $\tau_{\mathrm{PEI}}$.

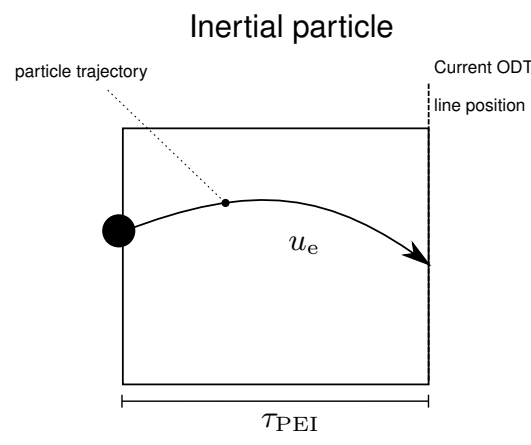

Tracer particle

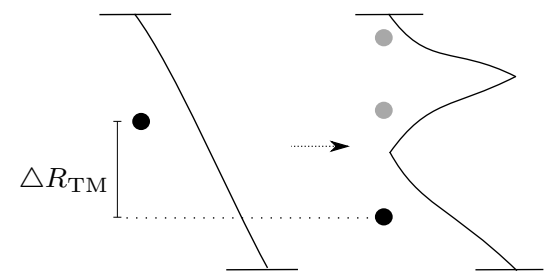

Figure 3. Example of re-integration of drag law (Eq. 9) over particle-eddy interaction time $\tau_{\text {PEI }}$. Eddy velocity $u_{\mathrm{e}}$ is defined as the tracer particle displacement $\triangle R_{\mathrm{TM}}$ by the triplet map divided by the eddy time scale $t_{e}$. For the displacement one (black circle) of three possible positions (grey circles) is chosen randomly.

Determining the radial gas phase velocity $u_{g, r}$ during the eddy time, the concept of the displacement of a mass-less tracer particle governed by the mapping method (4) is used. This method provides three possible tracer particle positions and a unique position is sampled randomly with a uniform distribution from those three possible ones. The final displacement $\triangle R_{T M}$, see Fig. 3, divided by the eddy time scale $t_{\mathrm{e}}$ defines the gas velocity during the PEl. As a next step the integral time scale has to be determine and therefore a so-called eddy box $\left[l \times l \times \beta_{p} t_{\mathrm{e}}\right]$, where $\beta_{p}$ is the model parameter for the PEI, is introduced. The PEI integration time $\tau_{\mathrm{PEI}}$ is given as the time the particle needs to exit the box. Therefore, the Stokes law is modified and analytically solved to find the earliest time when the particle leaves the space-time eddy box. 


\section{Test case}

\section{EXPERIMENTS}

For validation purposes the model is compared with experiments of Budilarto [1], which provide data for a constant air jet with a Reynolds number of 8400 and solid loadings of 0.5 and 1 . Also, different Stokes numbers are investigated by varying the single particle diameter. The jet exit has a diameter $D$ of $0.014224 \mathrm{~m}$. According to the Reynolds number, the jet exit velocity $u_{0}$ is $11.7 \mathrm{~m} / \mathrm{s}$. The particles used are glass beads with a density $\rho_{p}=2500$ $\mathrm{kg} / \mathrm{m}^{3}$ and with a number averaged diameter of $25 \mu \mathrm{m}$ and $75 \mu \mathrm{m}$. Their initial velocity depends on the coordinate framework of the simulation and is discussed in the following.

\section{SIMULATION SETUP}

As the initial jet profile in the ODT domain the following function is used

$$
u_{g, z}(r)=\frac{u_{g, z 0}}{2}\left[\left(1+\tanh \left(\frac{r-L_{1}}{\omega_{l}}\right)\right) \cdot\left(1-\frac{1}{2}\left(1+\tanh \left(\frac{r-L_{2}}{\omega_{l}}\right)\right)\right)\right] .
$$

$L_{1}$ and $L_{2}$ are the middle positions of the transitions and $\omega_{l}$ is the transition boundary layer width. Due to the fact that the axisymmetrical approach used to represent the jet flow configuraton in ODT is independent of the angular direction, the ODT line volume is determined by the square of the domain length. Thus, the solid loading, which is the ratio between particle-phase and gas-phase mass, is computed for the square of the jet diameter $D$. The initial particle positions are exponentially distributed over the nozzle exit. The initial velocities are based on the experimental data in [1]. The initial properties for the particle-phase in the ODT simulation are summarized in Tab. 1. This study uses 512 ODT realizations, which Sun et al. [12] reported as sufficient to capture stationary statistics.

Table 1. Initial particle-phase properties of particle diameter $d_{p}$, solid loading $s l$, axial velocity $u_{p, 0}$ number of parcels (particle clouds) $n_{p}$ and representing particles per parcel $N$, for a particle density of $2500 \mathrm{~kg} / \mathrm{m}^{3}$.

\begin{tabular}{c|cccc}
\hline$d_{p}(\mu \mathrm{m})$ & $s l$ & $u_{p, 0}(\mathrm{~m} / \mathrm{s})$ & $n_{p}$ & $N$ \\
\hline 25 & 0.5 & 11.324 & 310 & 9500 \\
& 1 & 11.205 & 310 & 19000 \\
70 & 0.5 & 9.664 & 300 & 450 \\
& 1 & 9.474 & 300 & 900
\end{tabular}

\section{Results}

SINGLE PHASE FLOW

Considering the assessment of gas-particle interaction, the first important step is to achieve an agreement between ODT simulations and experimental data for the single-phase case without particles. The ODT parameters are equal to the ones reported in [3] due to the same flow configuration, i.e. for the viscous penalty parameter we have $Z=400$ and for the eddy frequency parameter $C=12$. These parameters result in an overall agreement between ODT results and experimental data for the mean axial velocity and the velocity fluctuation along the centerline and for the mean axial velocity in radial direction for $x / D=5,10$ and 15 as shown in Fig. 4a and Fig. 4b, respectively. Axial and radial positions are both normalized by the jet diameter $D$ and axial velocities and fluctuations by the

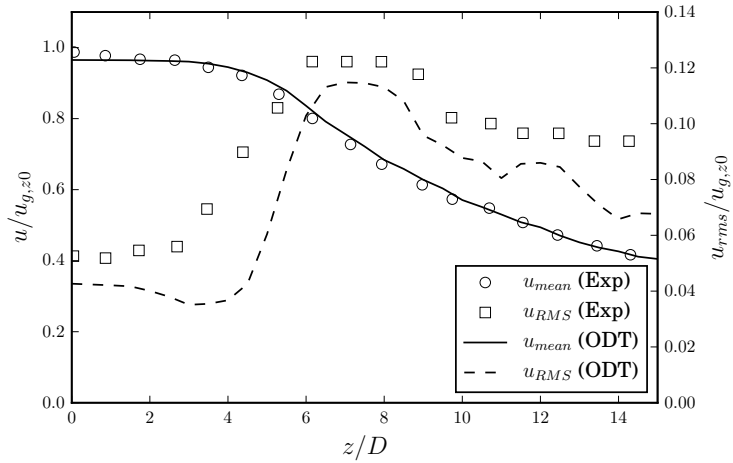

(a) Mean axial velocity along centerline

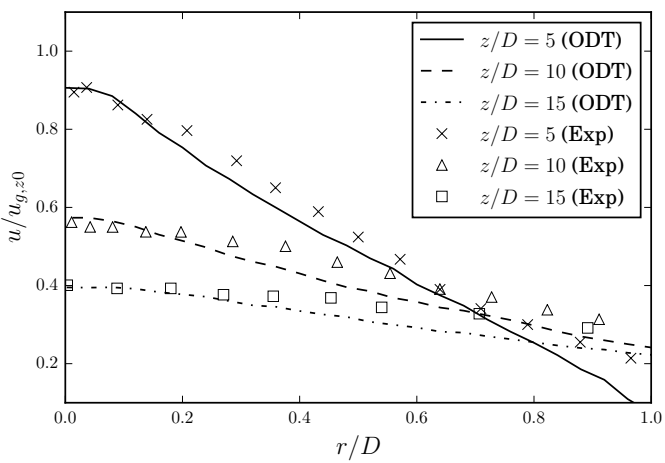

(b) Mean axial velocity profiles in radial direction

Figure 4. Axial velocity profiles of gas phase.

nozzle exit velocity $u_{g, z 0}$. Fig. 4a shows the same axial velocity decay along the centerline as the experimental data. The characteristics of the velocity fluctuations is also well captured. Additionally, the axial velocities in radial direction show good agreement with the experimental data, as shown in Fig. 4b. 
TWO-PHASE FLOW

For the two-phase flow simulations the particle-eddy interaction parameter $\beta_{p}$ is set to 0.008 based on previous parameter studies which are not discussed here. In the following the development of the mean axial centerline velocity and of the corresponding velocity fluctuation along the centerline is presented for different solid loadings in Fig. 5 and Fig. 6, respectively. Axial positions are normalized by the jet diameter $D$ and axial velocities and fluctuations by the nozzle exit velocity $u_{g, z 0}$. Fig. 5 shows that ODT is capturing the trend of the experimental data

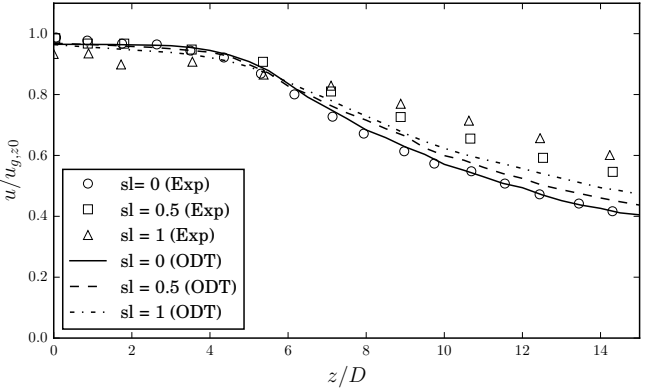

(a) $d_{p}=70 \mu \mathrm{m}$

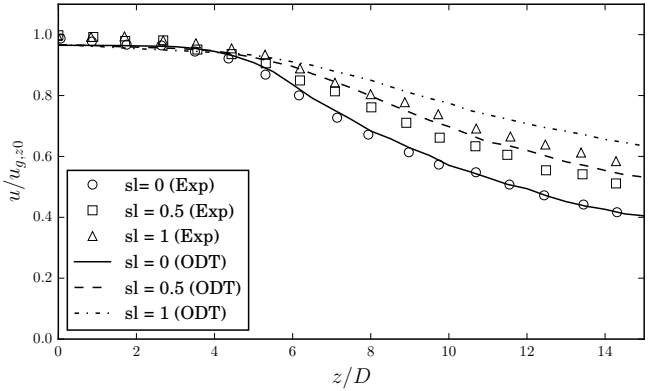

(b) $d_{p}=25 \mu \mathrm{m}$

Figure 5. Mean axial velocity along centerline with different solid loadings.

reasonably well. For a diameter of $70 \mu \mathrm{m}$ the differences in the ODT results for different solid loadings are smaller than observed in the experiments, but ODT is capturing the trend of increasing gas phase velocities/momentum for $z / D>5 D$ with increasing solid loading. Also, the velocity decrease till $z / D \sim 5 D$ caused by the higher momentum penalty for $s l=1$ can be observed. In Fig. $5 \mathrm{~b}$ the deviations between ODT and experiments for the smaller particles with $d_{p}=25 \mu \mathrm{m}$ are smaller compared to the particles with $d_{p}=70 \mu \mathrm{m}$ in Fig. $5 \mathrm{a}$ and a slight over-prediction can be seen.

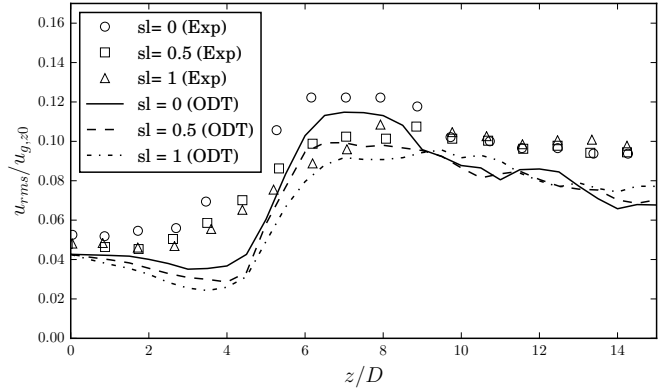

(a) $d_{p}=70 \mu \mathrm{m}$

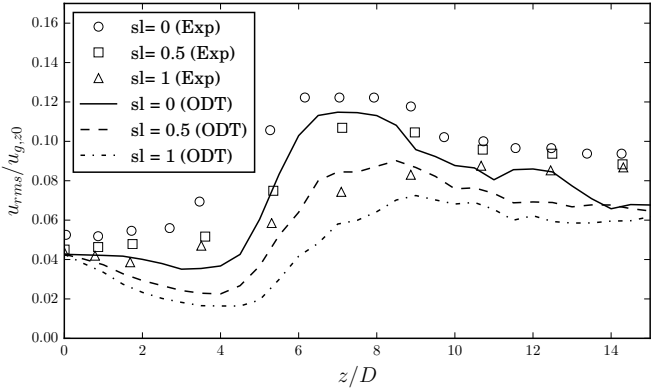

(b) $d_{p}=25 \mu \mathrm{m}$

Figure 6. Mean axial velocity fluctuation along centerline with different solid loadings

Another important indicator for turbulence modulation is the behavior of the turbulent velocity fluctuations for different solid loadings. The effect of turbulence suppression caused by solid particles is well shown in Fig. 6a and Fig. $6 \mathrm{~b}$. Both figures show a decrease of the velocity fluctuations with increasing solid loading and an enhanced damping of turbulence for the smaller particles.

\section{Conclusions}

The objective of this study was to extend the existing ODT model described in [12] by using a spatial approach in cylindrical coordinates. For this purpose the momentum and energy exchange between the particle and gas phase during the deterministic advancement and during the particle-eddy interaction were modified. Also, a new formulation to compute the kernel coefficients were introduced. The presented results show that ODT is capable of capturing the characteristics of a particle-laden jet with respect to variations in the solid loading. Furthermore, the results of Sun [12] could be reproduced without the need to transform the temporal coordinate. Additional extensions, e.g. considering droplet collision and coalescence, are planned for the near future. These model extensions are the base for the future goal to get a better understanding of the underlying physics of particle/dropletturbulence interaction. The scope for ODT will be fundamental investigations in parameter ranges which are not accessible using DNS or high resolution LES. 


\section{Nomenclature}

$d_{p} \quad$ particle diameter [m]

$D \quad$ jet diameter [m]

$m \quad$ mass $[\mathrm{kg}]$

$r \quad$ radial coordinate [m]

$u_{i} \quad$ i-th velocity component [m/s]

$z \quad$ axial coordinate $[\mathrm{m}]$

$\rho \quad$ density $\left[\mathrm{kg} / \mathrm{m}^{3}\right]$

Subscripts

$0 \quad$ initial

e eddy

$g \quad$ gas phase

$p \quad$ particle phase

PEI particle-eddy interaction

TM triplet map

\section{References}

[1] Budilarto, S. G., 2003, PhD thesis, Purdue Univeristy, USA.

[2] Clift, R., Grace, J. R. and Weber, M. E., 1978, Bubbles, drops and paticle, Academic Press, New York.

[3] Fistler, M., Lignell, D. O., Kerstein, A., Oevermann, M., Mai 15.-18. 2017, 29th ILASS-Americas.

[4] Geiss, S., Dreizler, A., Stojanovic, Z., Chrigui, M., Sadiki, A. and Janicka, J., 2004, Experiments in Fluids, 36 (2), pp. 344-354.

[5] Kerstein, A. R., 1999, Journal of Fluid Mechanics, 392, pp.277-334.

[6] Kerstein, A. R. and Ashurst, W. T., 2005, Physics of Fluid, 17-025107, pp.1-26.

[7] Lignell, D. O., Kerstein, A. R., Sun G. and Monson, E. I., 2013, Theoretical and Computational Fluid Dynamics, 27(3-4), pp. 273-295.

[8] Poelma, C., 2004, PhD thesis, TU Delft, Netherlands.

[9] Schmidt, J. R., Wendt, J. O. L., Kerstein, A. R.,2004, IUTAM Symposium on Computational Approaches to Disperse Multiphase Flow.

[10] Schmidt, J. R., Wendt, J. O. L., Kerstein, A. R.,2009, Journal of Statistical Physics, 37, pp. 233-257.

[11] Schreck, S. and Kleis, S., 1993, Journal of Fluid Mechanics, 249, pp. 665-688.

[12] Sun, G., Hewson, J. C., Lignell, D. O., 2017, Submitted to International Journal of Multiphase flow. 